\title{
A meshless stress analysis of nonhomogeneous materials using the triple-reciprocity boundary element method
}

\author{
Y. Ochiai \\ Department of Mechanical Engineering, Kinki University, Japan
}

\begin{abstract}
In general, internal cells are required to solve nonhomogeneous elastic problems using a conventional boundary element method (BEM). However, in this case, the merit of the BEM, which is the ease of data preparation, is lost. In this study, it is shown that two-dimensional nonhomogeneous elastic problems can be solved using the triple-reciprocity BEM without the use of internal cells. Young's modulus and Poisson's ratio are variable in nonhomogeneous elastic materials. The distribution of a fictitious body force generated by a nonhomogeneous material is interpolated using boundary integral equations. A new computer program was developed and applied to solve several problems.
\end{abstract}

Keywords: boundary element method, stress analysis, nonhomogeneous materials.

\section{Introduction}

Stress problems of nonhomogeneous materials can be solved by a conventional boundary element method (BEM) using internal cells for domain integrals [1].

In this case, however, the merit of the BEM, which is the ease of data preparation, is lost. Several countermeasures to prevent this loss have been considered. For example, Nowak and Neves proposed the conventional multiplereciprocity boundary element method (MRBEM). In the conventional MRBEM, the distribution of body forces must be given analytically, and fundamental solutions of higher order are used to make the solutions converge. Accordingly, this method is not suitable for the analysis of materials with nonhomogeneous elasticity. The dual-reciprocity BEM has been proposed for reducing the dimensionality of problems, which is an advantage of the BEM [2]. However, it is 
difficult to apply the dual-reciprocity BEM to nonhomogeneous problems. Sladek et al. [3] applied the local integral equation method to nonhomogeneous problems without internal cells. However, it was assumed that Poisson's ratio is constant. Xiao-Wei Gao et al. [4] applied the radial integral method to nonhomogeneous problems without internal cells. However, it was also assumed that Poisson's ratio was constant. Ochiai et al. [5] and Ochiai and Kobayashi [6] proposed the triplereciprocity BEM (improved multiple-reciprocity BEM) without using internal cells for elastoplastic problems. Using this method, a highly accurate solution can be obtained using only fundamental solutions of low order and with reduced need for data preparation. They applied the triple-reciprocity BEM without using internal cells to two-dimensional elastoplastic problems using initial strain formulations. Ochiai and Kobayashi [7] applied the triple-reciprocity BEM to thermo-elastoplastic problems with arbitrary heat generation and to threedimensional elastoplastic problems using initial strain formulations. Only the triple-reciprocity BEM and the local integral equation method have been applied to elastoplastic problems without internal cells.

In this study, the triple-reciprocity BEM is applied to two-dimensional nonhomogeneous elastic problems. Young's modulus and Poisson's ratio are variable in nonhomogeneous elastic materials. In this method, boundary elements and arbitrary internal points are used. The arbitrary distributions of a fictitious body force generated by a nonhomogeneous elastic material are interpolated using boundary integral equations and internal points. This interpolation corresponds to a thin plate spline. A new computer program is developed and applied to several nonhomogeneous elastic problems to clearly demonstrate the theory. This method is demonstrated to be efficient for calculation.

\section{Theory}

\subsection{Stress analysis of nonhomogeneous materials}

Denoting a displacement as $u_{i}$, the relationship between stress $\sigma_{i j}$ and strain $\varepsilon_{i j}$ is given by

$$
\sigma_{i j}=\lambda(p) \varepsilon_{k k} \delta_{i j}+2 \mu(p) \varepsilon_{i j},
$$

where $\lambda(p)$ and $\mu(p)$ are Lame's constants at point $p$. The relationship between displacement $u_{i}$ and strain $\varepsilon_{i j}$ is given by

$$
\varepsilon_{i j}=\frac{1}{2}\left(\frac{\partial u_{i}}{\partial x_{j}}+\frac{\partial u_{j}}{\partial x_{i}}\right)
$$

Denoting a body force as $F_{i}$, the following equation is obtained from Eq. (1) 


$$
\mu \nabla^{2} u_{i}+(\lambda+\mu) \frac{\partial}{\partial x_{i}} \frac{\partial u_{j}}{\partial x_{j}}+\frac{\partial \lambda}{\partial x_{i}} \frac{\partial u_{j}}{\partial x_{j}}+\frac{\partial \mu}{\partial x_{j}} \frac{\partial u_{j}}{\partial x_{i}}+\frac{\partial \mu}{\partial x_{j}} \frac{\partial u_{i}}{\partial x_{j}}+F_{i}=0
$$

In Eq. (3), the terms associated with a nonhomogeneous material are denoted a fictitious body force $b_{i}^{[1]}(q)$

$$
b_{i}^{[1]}(q)=\frac{\partial \lambda}{\partial x_{i}} \frac{\partial u_{j}}{\partial x_{j}}+\frac{\partial \mu}{\partial x_{j}} \frac{\partial u_{j}}{\partial x_{i}}+\frac{\partial \mu}{\partial x_{j}} \frac{\partial u_{i}}{\partial x_{j}} .
$$

The following boundary integral equation must be solved [1, 6-8]:

$$
c_{i j} u_{j}(P)=\int_{\Gamma}\left[u_{i j}^{[1]}(P, Q) p_{j}(Q)-p_{i j}(P, Q) u_{j}(Q)\right] d \Gamma+\int_{\Omega} u_{i j}^{[1]}(P, q) b_{j}^{[1]}(q) d \Omega,
$$

where $c_{i j}$ is the free-term coefficient. Moreover, $u_{j}$ and $p_{j}$ are the $j$ th components of the displacement and surface traction, respectively. Furthermore, $\Omega$ and $\Gamma$ are the analyzed domain and its boundary, respectively. Denoting the distance between the observation point and the loading point by $r$, Kelvin's solution $u_{i j}^{[1]}$ and $p_{i j}$ are given by

$$
\begin{gathered}
u_{i j}^{[1]}(p, q)=\frac{1}{8 \pi\left(1-v_{0}\right) G_{0}}\left[\left(3-4 v_{0}\right) \delta_{i j} \ln \left(\frac{1}{r}\right)+r,_{i} r,{ }_{j}\right], \\
p_{i j}(p, q)=\frac{-1}{4 \pi\left(1-v_{0}\right) r}\left\{\left[\left(1-2 v_{0}\right) \delta_{i j}+2 r_{,} r,{ }_{j}\right] \frac{\partial r}{\partial n}-\left(1-2 v_{0}\right)\left(r_{,} n_{j}-r,{ }_{j} n_{i}\right)\right\},
\end{gathered}
$$

where $v_{0}$ is Poisson's ratio and $G_{0}$ is the shear modulus at point $p$. Moreover, we set $v_{0}^{\prime}=v /(1+v)$ for the plane stress. The notation $n_{i}$ is used for the $i$ th component of the outward unit normal vector on $\Gamma$. Equation (5) has a domain integral.

\subsection{Interpolation of body force}

Interpolation is performed using boundary integrals to avoid the domain integral in Eq. (3) $[9,10]$. The two-dimensional distribution of the body force $b_{j}^{[1] S}(q)$ is interpolated using the integral equations to transform the domain integral into a boundary integral. The following equations are used for interpolation:

$$
\begin{aligned}
& \nabla^{2} b_{j}^{[1] S}(q)=-b_{j}^{[2] S}(q), \\
& \nabla^{2} b_{j}^{[2] S}(q)=-b_{j}^{[3] P}(q),
\end{aligned}
$$


where

$$
\nabla^{4} b_{j}^{[1] S}(q)=\sum_{m=1}^{M} b_{j}^{[3] P}\left(q_{m}\right) \delta\left(q-q_{m}\right)
$$

This interpolation is assumed for each body force $(j=x, y)$.

\subsection{Boundary integral equations used for interpolation}

The polyharmonic function $T^{[f]}$ satisfies the relationship

$$
\nabla^{2} T^{[\mathrm{f}+1]}(p, q)=T^{[f]}(p, q)
$$

Therefore, the function $T^{[f]}$ can be obtained as

$$
T^{[f+1]}=\int \frac{1}{r}\left(\int r T^{[f]} d r\right) d r .
$$

The polyharmonic function $T^{[f]}$ in two-dimensional problems is given by

$$
\begin{gathered}
T^{[f]}(p, q)=\frac{r^{2(f-1)}}{2 \pi 4^{f-1}[(f-1) !]^{2}}\left[\ln \left(\frac{1}{r}\right)+C_{q}+\operatorname{sgn}(f-1) \sum_{e=1}^{f-1} \frac{1}{e}\right] \\
=\frac{r^{2(f-1)}}{2 \pi[(2 f-2) ! !]^{2}}\left[\ln \left(\frac{1}{r}\right)+C_{q}+\operatorname{sgn}(f-1) \sum_{e=1}^{f-1} \frac{1}{e}\right],
\end{gathered}
$$

where $(2 f) ! !=2 f(2 f-2) \cdots 4 \cdot 2$ and $C_{q}$ is an arbitrary constant. $C_{q}=0$ can be assumed for the interpolation. The body force $b_{j}^{[1] S}(P)$ is given by Green's theorem and Eqs. (8) and (9) as

$$
\begin{aligned}
& c b_{j}^{[1] S}(P)=\sum_{F=1}^{2} \int_{\Gamma}\left\{T^{[f]}(P, Q) \frac{\partial b_{j}^{[f] S}(Q)}{\partial n}-\frac{\partial T^{[f]}(P, Q)}{\partial n} b_{j}^{[f] S}(Q)\right\} d \Gamma \\
& -\sum_{m=1}^{M} T_{i}^{[2]}(P, q) b_{j(m)}^{[3] P}(q),
\end{aligned}
$$

where $c=0.5$ on the smooth boundary and $c=1$ in the domain. The curvature of the body force distribution $b_{j}^{[2] S}(P)$ is given by Green's theorem and Eq. (9) as

$$
\begin{gathered}
c b_{j}^{[2] S}(P)=\int_{\Gamma}\left\{T^{[f]}(P, Q) \frac{\partial b_{j}^{[f] S}(Q)}{\partial n}\right. \\
\left.-\frac{\partial T^{[f]}(P, Q)}{\partial n} b_{j}^{[f] S}(Q)\right\} d \Gamma+\sum_{m=1}^{M} T_{i}^{[1]}(P, q) b_{j(m)}^{[3] P}(q) .
\end{gathered}
$$


The fictitious body force $b_{i}^{[1] S}$ is known from Eq. (4). Then, the boundary unknowns $\partial b_{j}^{[f] S} / \partial n$ and the unknowns $b_{j}^{[3] P}\left(q_{m}\right)$ at interior points can be calculated by simultaneous solution of the integral equations (14) and (15) with the latter being collocated at $M$ interior points and assuming $b_{i}^{[2] S}(Q)=0$.

\subsection{Triple-reciprocity boundary element method}

The functions $u_{i j}^{[f]}$ are defined as

$$
\nabla^{2} u_{i j}^{[f+1]}(p, q)=u_{i j}^{[f]}(p, q)
$$

Using Eqs. (8), (9), (11) and (16), and Green's second identity, Eq. (5) becomes

$$
\begin{gathered}
c_{i j} u_{j}(P)=\int_{\Gamma}\left[u_{i j}^{[1]}(P, Q) p_{j}(Q)-p_{i j}(P, Q) u_{j}(Q)\right] d \Gamma \\
+\sum_{f=1}^{2}(-1)^{f} \int_{\Gamma}\left\{\frac{\partial u_{i j}^{[f+1]}(P, Q)}{\partial n} b_{j}^{[f] S}(Q)-u_{i j}^{[f+1]}(P, Q) \frac{\partial b_{k}^{[f] S}(Q)}{\partial n}\right\} d \Gamma \\
+\sum_{m=1}^{M} u_{i j}^{[3]}(P, q) b_{j(m)}^{[3] P}(q)
\end{gathered}
$$

From Eq. (17), the gradient of the displacement is obtained as

$$
\begin{gathered}
\frac{\partial u_{j}(P)}{\partial x_{k}}=\int_{\Gamma}\left[\frac{\partial u_{i j}^{[1]}(P, Q)}{\partial x_{k}} p_{j}(Q)-\frac{\partial p_{i j}(P, Q)}{\partial x_{k}} u_{j}(Q)\right] d \Gamma \\
+\sum_{f=1}^{2}(-1)^{f} \int_{\Gamma}\left\{\frac{\partial^{2} u_{i j}^{[f+1]}(P, Q)}{\partial x_{k} \partial n} b_{j}^{[f] S}(Q)-\frac{\partial u_{i j}^{[f+1]}(P, Q)}{\partial x_{k}} \frac{\partial b_{k}^{[f] S}(Q)}{\partial n}\right\} d \Gamma \\
+\sum_{m=1}^{M} \frac{\partial u_{i j}^{[3]}(P, q)}{\partial x_{k}} b_{j(m)}^{[3] P}(q)
\end{gathered}
$$

The higher-order Kelvin's solution $u_{i j}^{[f]}(p, q)$ is defined as

$$
u_{i j}^{[f]}=\frac{-1}{2(1-v) G} T^{[f+1]},_{i j}+\frac{\delta_{i j} T^{[f+1]}, k k}{G} .
$$


90 Boundary Elements and Other Mesh Reduction Methods XXXVII

Substituting Eq. (13) into Eq. (19), we obtain

$$
\begin{aligned}
u_{i j}^{[f]}=\frac{r^{2(f-1)}}{4 \pi[(2 f) ! !]^{2}(1-v) G}\left\langle\left[4 f(f-1) F_{2}+2(2 f-1)\right] r,{ }_{i} r,{ }_{j}\right. \\
\left.\left.+[2 f(1-4 f+4 f v)] F_{2}+1-8 f(1-v)\right] \delta_{i j}\right\rangle,
\end{aligned}
$$

where

$$
\begin{gathered}
C_{q}=\frac{7-8 v}{2(3-4 v)} \\
F_{2}=\ln (r)-C_{q}-\operatorname{sgn}(f-1) \sum_{e=2}^{f} \frac{1}{e} .
\end{gathered}
$$

From Eq. (20), the derivatives in Eq. (18) are obtained as follows:

$$
\begin{aligned}
& \frac{\partial u_{i j}^{[f]}}{\partial n}=\frac{r^{2 f-3}}{2 \pi\{(2 f) ! !\}^{2}(1-v) G}\left\langle\left\{ 2\left[2 f(f-1) F_{2}+\left(3 f^{2}-6 f+2\right)\right] r,{ }_{i} r,{ }_{j}\right.\right. \\
& \left.+\left\{2 f(f-1)[1-4(1-v)] F_{2}+(2 f-1)-4(1-v) f(3 f-2)\right\} \delta_{i j}\right\} r,{ }_{s} n_{s} \\
& \left.+\left[2 f(f-1) F_{2}+(2 f-1)\right]\left(n_{i} r,{ }_{j}+n_{j} r{ }_{i}\right)\right\rangle \\
& \frac{\partial p_{i j}}{\partial x_{s}}=\frac{1}{4 \pi(1-v) r^{2}}\left\{2 \frac{\partial r}{\partial n}\left[\delta_{i s} r,{ }_{j}+\delta_{j s} r,{ }_{i}-r,{ }_{s}\left((1-2 v) \delta_{i j}+4 r,{ }_{i} r,{ }_{j}\right)\right]\right. \\
& +n_{s}\left[(1-2 v) \delta_{i j}+2 r,{ }_{i} r,{ }_{j}\right]-n_{j}(1-2 v)\left(\delta_{i s}-2 r,{ }_{i} r,{ }_{s}\right) \\
& \left.+n_{i}(1-2 v)\left(\delta_{j s}-2 r,{ }_{j} r,{ }_{s}\right)\right\} \text {, } \\
& \frac{\partial u_{i j}^{[f]}}{\partial x_{s}}=\frac{r^{2 f-3}}{2 \pi\{(2 f) ! !\}^{2}(1-v) G}\left\langle\left\{ 2\left[2 f(f-1) F_{2}+\left(3 f^{2}-6 f+2\right)\right] r,{ }_{i} r,{ }_{j}\right.\right. \\
& \left.+\left\{2 f(f-1)[1-4(1-v)] F_{2}+(2 f-1)-4(1-v) f(3 f-2)\right\} \delta_{i j}\right\} r,{ }_{s} \\
& \left.+\left[2 f(f-1) F_{2}+(2 f-1)\right]\left(\delta_{s i} r,{ }_{j}+\delta_{s j} r,{ }_{i}\right)\right\rangle,
\end{aligned}
$$




$$
\begin{aligned}
& \frac{\partial u_{i j}^{[f]}}{\partial x_{s} \partial x_{k}} n_{s}=\frac{r^{2 f-4}}{2 \pi\{(2 f) ! !\}^{2}(1-v) G}\left\langle\left[ 2 f(f-1)(4 f-9) F_{2}\right.\right. \\
& \left.+2\left(3 f^{3}-14 f^{2}+19 f-6\right)\right] r,{ }_{i} r,{ }_{j} r,{ }_{k} r,{ }_{s} n_{s} \\
& +\left\{(2 f-3) 2 f(f-1)[1-4(1-v)] F_{2}+\left(6 f^{2}-10 f+3\right)\right\} \delta_{i j} r,{ }_{k} r,{ }_{s} n_{s} \\
& +\left[(2 f-3) 2 f(f-1) F_{2}+\left(6 f^{2}-10 f+3\right)\right] r,{ }_{s} n_{s}\left(\delta_{k i} r,{ }_{j}+\delta_{k j} r{ }_{i}\right) \\
& +2\left[2 f(f-1) F_{2}+\left(3 f^{2}-6 f+2\right)\right]\left(n_{i} r,{ }_{j}+n_{j} r,{ }_{i}\right) r,{ }_{k} \\
& +2\left[2 f(f-1) F_{2}+\left(3 f^{2}-6 f+2\right)\right] r,{ }_{i} r,{ }_{j} n_{k} \\
& +\left\{2 f(f-1)[1-4(1-v)] F_{2}+(2 f-1)-4(1-v) f(3 f-2)\right\} \delta_{i j}\left(n_{k}-n_{s} r,{ }_{s} r,_{k}\right) \\
& \left.+\left[2 f(f-1) F_{2}+(2 f-1)\right]\left[\delta_{k i}\left(n_{j}-n_{s} r,{ }_{s} r,{ }_{j}\right)+\delta_{k j}\left(n_{i}-n_{s} r,{ }_{s} r,_{i}\right)\right]\right\rangle .
\end{aligned}
$$

In the same manner, the internal stress can be obtained. The relationship between the displacement and stress is given as

$$
\sigma_{i j}=\frac{2[1-v(p)] G(p)}{[1-2 v(p)]} \delta_{i j} u_{k},{ }_{k}+G(p)\left(u_{i},{ }_{j}+u_{j},{ }_{i}\right) .
$$

Substituting the values of Eq. (18) into Eq. (27), the internal stress $\sigma_{i j}$ can be obtained.

\subsection{In the case of composite materials}

In the case of composite materials or a layer structure, the distribution of Lame's constant $\lambda_{1}(q)$ is continuous but not smooth as shown in Figure 1. The fictitious body force $b_{j}^{[1]}$ in Eq. (6) has a discontinuous value of $b_{j}^{[1] D}$. Therefore, the following equations can be used to interpolate the fictitious body force:

$$
\begin{gathered}
\nabla^{2} b_{j}^{[1] S}=-b_{j}^{[2] S}-b_{j}^{[2] D} \\
\nabla^{2} b_{j}^{[2] S}=-\sum_{m=1}^{M} b_{j}^{[3] P}\left(q_{m}\right) .
\end{gathered}
$$

As shown in Figure 1(a), the distribution of Lame's constant $\lambda_{1}(q)$ has a discontinuous value $\lambda_{1}^{D}$. The following equation can be used for the distribution of $\lambda_{1}(q)$ : 


$$
\lambda_{1}=\lambda_{1}^{S}+\lambda_{1}^{D}
$$

where $\lambda_{1}^{D}=\lambda^{[1]}-\lambda^{[2]}$. As shown in Figure 1(b), we can obtain the fictitious line force $b_{j}^{[1] L}(q)$ in Eq. (4), which is generated from the discontinuous Lame's constant, using $B \rightarrow 0$. We can express $b_{j}^{[1] L}(q)$ as

$$
\begin{gathered}
b_{j}^{[1] L}(q)=\nabla \lambda_{1}^{S} \cdot \nabla u=\lim _{B \rightarrow 0}\left(\lambda^{[2]}-\lambda^{[1]}\right)\left(q^{[1]}+q^{[2]}\right) / 2 \\
=\frac{\left(\lambda^{[2]}-\lambda^{[1]}\right)\left(q^{[1]}+q^{[2]}\right)}{2} \delta\left(q-q_{D}\right), \\
b_{j}^{[1]}=-b_{j}^{[1] S}+b_{j}^{[1] L},
\end{gathered}
$$

where $q^{[i]}=\partial u^{[i]} / \partial n^{\prime}$. From Eq. (30), we can obtain the following boundary integral equation.

$$
\begin{gathered}
c_{i j} u_{j}(P)=\int_{\Gamma}\left[u_{i j}^{[1]}(P, Q) p_{j}(Q)-p_{i j}(P, Q) u_{j}(Q)\right] d \Gamma+\int_{\Gamma} u_{i j}^{[1]}(P, Q) b_{j}^{[1] L}(q) d \Gamma^{\prime} \\
+\sum_{f=1}^{2}(-1)^{f} \int_{\Gamma}\left\{\frac{\partial u_{i j}^{[f+1]}(P, Q)}{\partial n} b_{j}^{[f] S}(Q)-u_{i j}^{[f+1]}(P, Q) \frac{\partial b_{k}^{[f] S}(Q)}{\partial n}\right\} d \Gamma \\
+\sum_{m=1}^{M} u_{i j}^{[3]}(P, q) b_{j(m)}^{[3] P}(q) .
\end{gathered}
$$

The boundary $\Gamma^{\prime}$ is the interface between two materials with different properties.

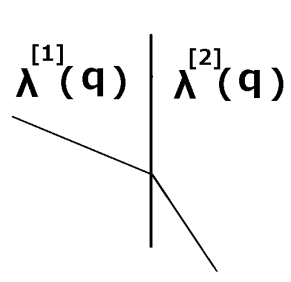

(a) Continuous case

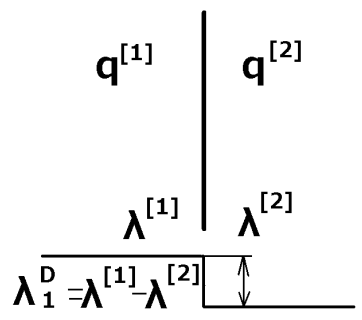

(b) Two different materials

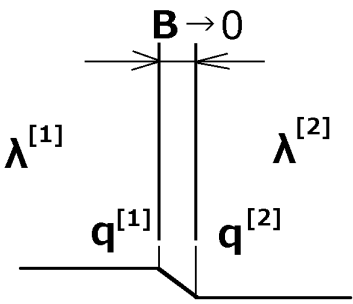

(c) Concept of fictitious force

Figure 1: Non-smooth distributions of Lame's constant $\lambda$. 


\section{Numerical examples}

To verify the accuracy of the present method, the stresses are computed in a rectangular plate made of a functionally graded material as shown in Figure 2. The Length $L$ and width of the plate are $100 \mathrm{~mm}$ and $40 \mathrm{~mm}$, respectively. The plate is restricted in the $x$ direction at $x=0$ and the load is $p_{0}=100 \mathrm{~N} / \mathrm{mm}$ at $x=L$.

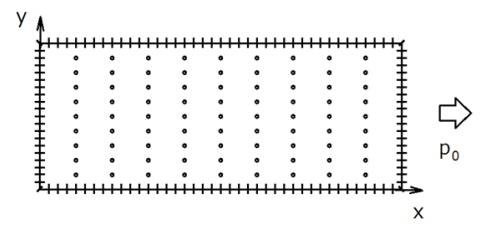

Figure 2: Nonhomogeneous rectangular plate (boundary elements and internal points).

Young's modulus $E(x)$ for the plate is given by

$$
E(x)=A \sin \left(\frac{\pi x}{L}\right)+B,
$$

where $A=-100 \mathrm{GPa}, \quad B=200 \mathrm{GPa}$ and Poisson's ratio is $v=0.3$. Figure 3 shows the displacements distribution with the exact solution. In the case of a linear change in Young's modulus, the displacement is obtained. Here, Young's modulus $E(x)$ is given by

$$
E(x)=A \frac{x}{L}+B,
$$

where $A=-100 \mathrm{GPa}, \quad B=200 \mathrm{GPa}$ and Poisson's ratio is $v=0.3$. Figure 4 shows the displacements distribution with the exact solution.

The displacement and stress in a nonhomogeneous square plate of length $L=100 \mathrm{~mm}$, as shown in Figure 5, are next calculated. The square plate is restricted in the $x$ direction at $x=0$ and the load is $p_{0}=100 \mathrm{~N} / \mathrm{mm}$ at $x=L$. Young's modulus $E(x)$ is given by

$$
E(x, y)=A \sin \left(\frac{\pi x}{L}\right) \sin \left(\frac{\pi y}{L}\right)+B,
$$

where $A=-100 G P a, B=200 G P a$ and Poisson's ratio is $v=0.3$. Figure 6 shows the stress distributions with the FEM solutions. In the next example, Poisson's ratio is variable and given by

$$
v(x, y)=A \sin \left(\frac{\pi x}{L}\right) \sin \left(\frac{\pi y}{L}\right)+B,
$$

where $A=-0.2, B=0.4$, and Young's modulus is $E=200 \mathrm{GPa}$. Figure 7 shows the displacement distribution with the FEM solution. 
94 Boundary Elements and Other Mesh Reduction Methods XXXVII

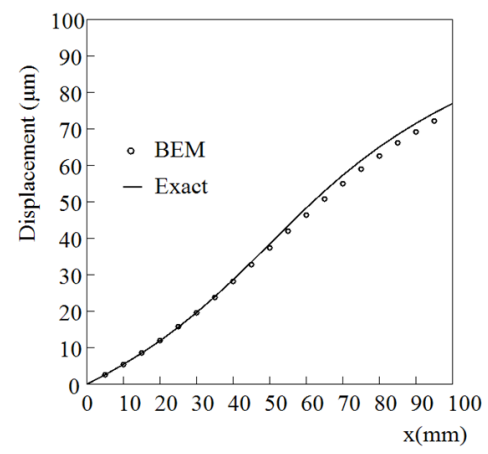

Figure 3: Displacement distribution of rectangular plate.

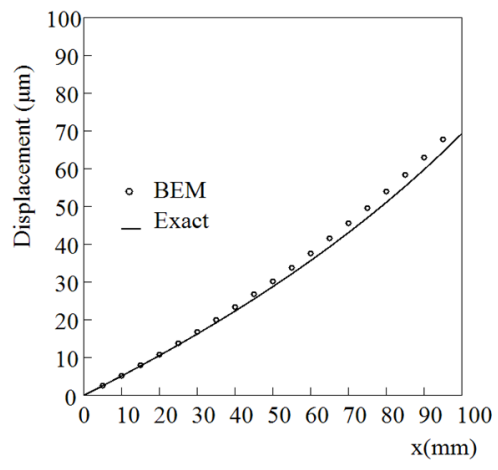

Figure 4: Displacement distribution of rectangular plate.

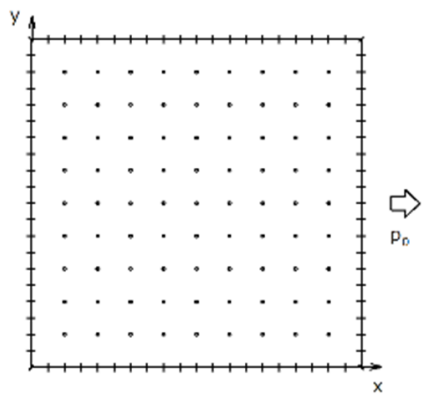

Figure 5: Square plate (boundary elements and internal points). 


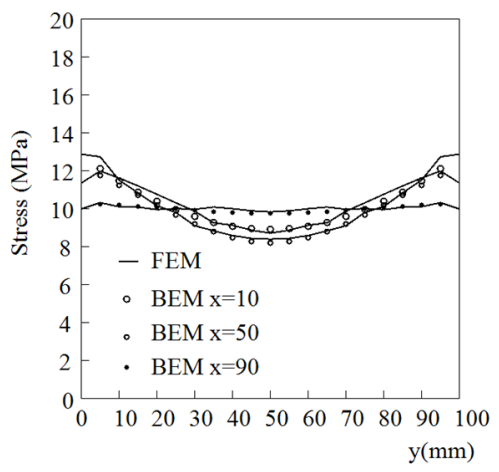

Figure 6: Stress distributions in square plate.

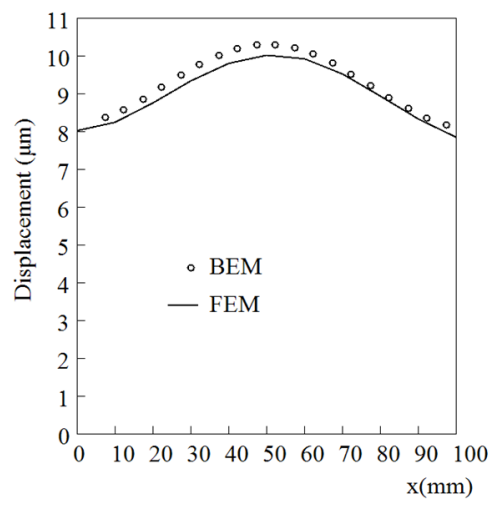

Figure 7: Displacement distribution of square region with variable Poisson's ratio.

Next, a laminated rectangular plate of length $L=10 \mathrm{~mm}$, as shown in Figure 8 , is forced in the $x$ direction of $p_{0}=100 \mathrm{~N} / \mathrm{mm}$. Here, Young's modulus $E(x)$ is given by

$$
E(x)=\left\{\begin{array}{cc}
200 & 0 \leq x \leq 3 \\
100 & 3 \leq x \leq 7 \\
200 & 7 \leq x \leq 10
\end{array}\right.
$$

and the width of the plate is $4 \mathrm{~mm}$. The rectangular plate is restricted in a $\mathrm{x}$ direction at $x=0$, and load is $p_{0}=100 \mathrm{~N} / \mathrm{mm}$ at $x=L$. Figure 9 shows the displacements distribution with the exact solution. 
The stress distributions in composite square plate of a length $L$ as shown in Figure 10 were also calculated. Young's modulus in the square region is $\mathrm{E}=200 \mathrm{GPa}$, and Young's modulus in the circular region is $0 \mathrm{GPa}, 100 \mathrm{GPa}$ or $400 \mathrm{GPa}$. The square plate is restricted in the $\mathrm{x}$ direction at $x=0$, and the load is $p_{0}=100 \mathrm{~N} / \mathrm{mm}$ at $x=L$. Figure 11 shows the displacements with the exact solutions.

The stress distribution in a circular plate with a hole made of functionally graded material, as shown in Figure 12, was next calculated. The outer radius is $R_{O}=30 \mathrm{~mm}$, the inner radius is $R_{I}=10 \mathrm{~mm}$ and the internal pressure is $P=100$ $\mathrm{MPa}$. Young's modulus $E(r)$ is given by

$$
E(r)=A\left(R_{0}-r\right)\left(r-R_{I}\right)+B
$$

where $A=-10 \mathrm{GPa}, \quad B=200 \mathrm{GPa}$, and Poisson's ratio is $v=0.3$. Figure 13 shows the displacement with the exact solution and Figure 14 shows the strain distribution with the exact solution.

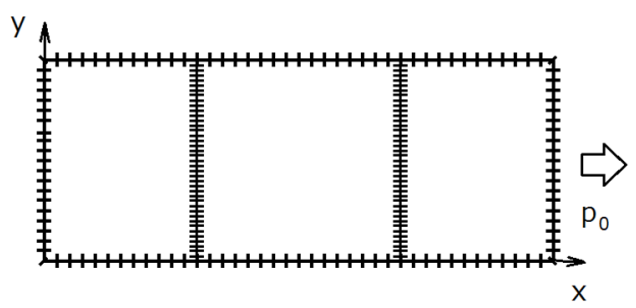

Figure 8: Boundary elements and internal elements of laminated plate.

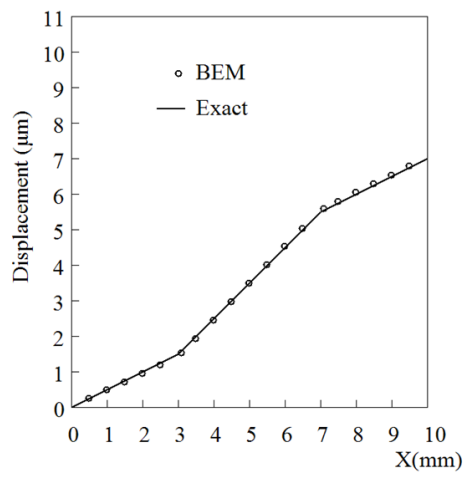

Figure 9: Displacement distribution of laminated plate. 


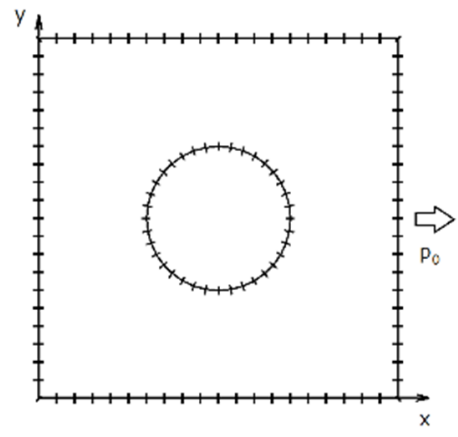

Figure 10: Square plate with a circular region.

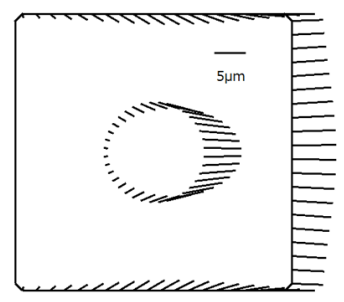

$\mathrm{E}=0 \mathrm{GPa}$

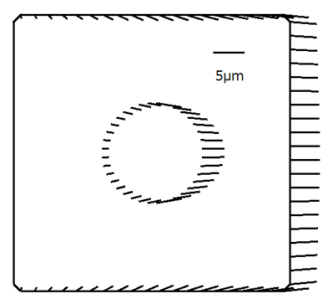

$\mathrm{E}=100 \mathrm{GPa}$

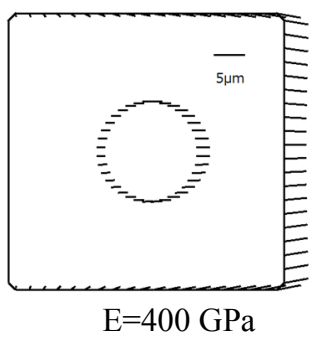

$\mathrm{E}=400 \mathrm{GPa}$

Figure 11: Displacements of plates with a circular region.

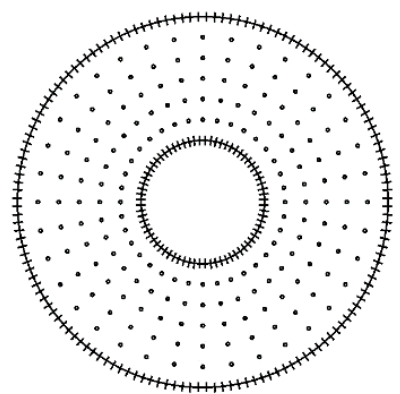

Figure 12: Nonhomogeneous circular plate with a hole. 


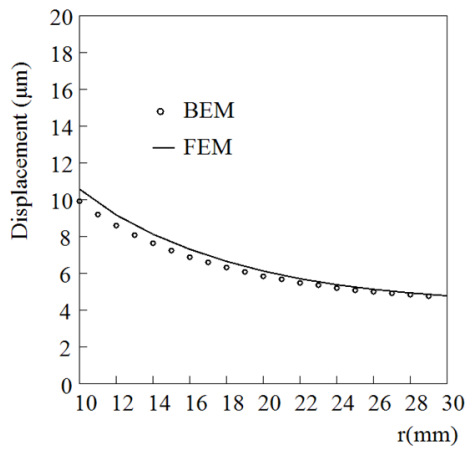

Figure 13: Displacement of circular plate.

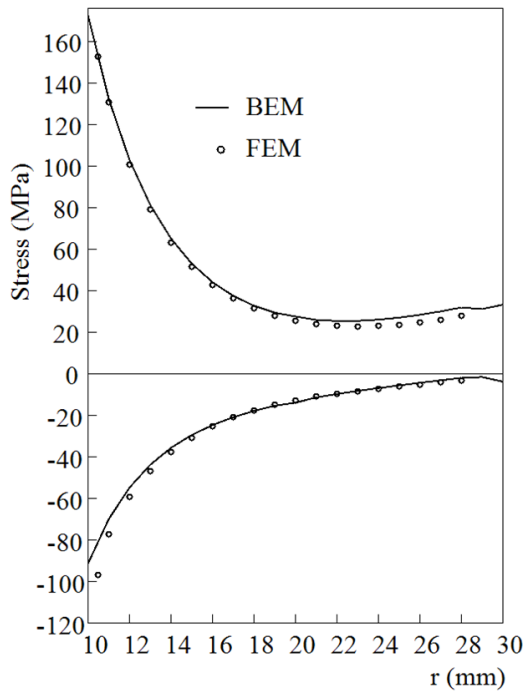

Figure 14: Stress distribution of circular plate.

\section{Conclusions}

The triple-reciprocity boundary element method was developed for the solution of two-dimensional elastic problems in nonhomogeneous solid bodies. A fictitious body force caused by a nonhomogeneous materials is used in its formulation. The sets of fundamental solutions for this analysis were derived. The main advantages of this method is that the use of internal cells is completely avoided. Thus, the merit of the BEM, which is the easy preparation of data, is retained because internal cells are not necessary. The numerical results provided for several examples illustrate the effectiveness and accuracy of the proposed method. 


\section{References}

[1] Brebbia, C. A., Telles, J. C. F. and Wrobel, L. C., Boundary Element Techniques-Theory and Applications in Engineering, (1984) SpringerVerlag, pp. 252-266.

[2] Partridge, P. W., Brebbia, C.A. and Wrobel, L. C. , The Dual Reciprocity Boundary Element Method (1992), Computational Mechanics Publications, pp. 223-253.

[3] Sladek J., Sladek V. and Atluri S. N., Local boundary integral equation (LBIE) method for solving problems of elasticity with nonhomogeneous material properties, Computational mechanics 2000; 24: pp. 456-462.

[4] Xiao-Wei Gao, Ch. Zhang and L. Guo, "Boundary-only element solution of 2D and 3D nonlinear and nonhomogeneous problems", Engineering Analysis with Boundary Elements, Vol. 31 (2007), pp. 974-982.

[5] Ochiai, Y., Nishitani, H. and Sekiya, T., "Stress Analysis with Arbitrary Body Force by Boundary Element Method", Engineering Analysis with Boundary Elements, Vol. 17 (1996), pp. 295-302.

[6] Ochiai, Y. and Kobayashi, T., "Initial Strain Formulation without Internal Cells for Elastoplastic Analysis by Triple-Reciprocity BEM", International Journal for Numerical Methods in Engineering, Vol. 50 (2001), pp. 18771892.

[7] Ochiai, Y. and Kobayashi, T., "Initial Stress Formulation for Elastoplastic Analysis by Improved Multiple-Reciprocity Boundary Element Method", Engineering Analysis with Boundary Elements, Vol. 23 (1999), pp. 167173.

[8] Ochiai, Y. and Sladek, V., "Numerical Treatment of Domain Integrals without Internal Cells in Three-Dimensional BIEM Formulations", CMES (Computer Modeling in Engineering \& Sciences), Vol. 6, No. 6 (2004), pp. 525-536.

[9] Ochiai, Y. and Yasutomi, Z., "Improved Method Generating a Free-Form Surface Using Integral Equations", Computer-Aided Geometric Design, Vol. 17, No. 3 (2000), pp. 233-245.

[10] Ochiai, Y., "Three-Dimensional Thermal Stress Analysis by TripleReciprocity Boundary Element Method", International Journal for Numerical Methods in Engineering, Vol. 63, No. 12 (2005), pp. 1741-1756. 